\title{
Evaluating brain signal of patients with mild Alzheimer in order to early separation of them from normal individuals
}

\author{
Fereydoon Naseri \\ MS.C in Biomedical Engineering, Department of Electrical and Computer Engineering, Shiraz University, Shiraz, Iran
}

fereydoon-naseri@shirazu.ac.ir

\section{Article history:}

Received date: 12 August, 2019

Review date: 3 October 2019

Accepted date:13 November 2019

Keywords:

Alzheimer, brain signals, wavelet

\begin{abstract}
The purpose and incentive of this study is designing and providing a system for identifying mild Alzheimer. Alzheimer can be identified by losing neurons synapses in some brain regions, necrotic brain cells in different parts of the nervous system, creating globular form of protein structures called senile plaques outside of neurons in some brain regions and filamentous protein structures called spiral coils in the cell body of neurons. FlexComp is a device used to record brain signals. Two channels of $F z$ and $P z$ are used for the reference of electrode ear to record the signals. Brain signals were taken from individual with open eyes for 2 minutes and 2 minutes blindfolded. Best results were extracted in delta rf open oz beta coherence close with close " wavelet Alpha sig close $f z$ wavelet theta sig open oz g FD sig open $f z$ modes..
\end{abstract}

Please cite this article as: Fereydoon Naseri. 2019. Evaluating brain signal of patients with mild Alzheimer in order to early separation of them from normal individuals. SRPH Journal of Fundamental Sciences and Technology, 1(1), 1-10

\section{Introduction:}

Alzheimer's is the most common form of dementia. The symptoms of the disease are beginning with loss of keeping information particularly short term memory in old age and gradually with losing time detection, depression, loss of speech, withdrawal and eventually it ends with death from respiratory distress. Death occurs after five to ten years from the onset of symptoms; but the disease has already started about twenty years before symptoms appear. Alois Alzheimer in 1906 could provide scientific definition of the after 20 years of study. This disease was named Alzheimer to appreciate a lifetime of research of Alois Alzheimer. Alzheimer is known as aging disease, in fact is something more than a simple forgetfulness (Güntekin et al, 2008). Alzheimer is a degenerative and rationalization progressive disease is usually seen in older people. Obvious symptoms of this disease are loss of memory, judgment and reasoning, and changes in the person's behavior. Alzheimer can be identified by losing neurons synapses in some brain regions, necrotic brain cells in different parts of the nervous system, creating globular form of protein structures called senile plaques outside of neurons in some brain regions and filamentous protein structures called spiral coils in the cell body of neurons and memory impairment is progresses and creates gradually. Initially, memory impairment is limited to recent events and learning but gradually old memories are getting damaged. The patient forgets the answer of a question that he asked a few moments ago and asks the same question again. He loses his stuff and do not remember where he put them. He has problem in buying goods and paying them and cannot handle his financial affairs. Gradually he has problem in identifying friends and family and cannot remember their names. He has problem in finding his routes and path and if he gets out of home alone, he get lost. Alzheimer occurs with brain atrophy and loss of some brain neurons and it is causing changes in behavior and performance of individuals. Usually, analysis of the brain is causing brain dysfunction and has its effect on different performance parts (Chapman et al, 2007). Therefore, for the treatment of Alzheimer we require a process to compensate the disorders resulting from brain atrophy and loss of neurons in the brain. As we know, the process of making neurons in human brain stops at the age of 2 years old and all the changes that occur in the brain after this age onwards are due to changes in the relationship between synapses. Therefore, finding a process to compensate for lost neurons, by increasing synaptic connections in healthy neurons can 
be used as a suitable solution for the treatment of Alzheimer. But unfortunately so far there is no definitive solution for the treatment of Alzheimer. The methods currently are used for the treatment of Alzheimer are based on methods for maintaining the patient status and preventing further weakening of the brain and loss of more brain neurons and improving mild effects of disease and behavioral approaches toward Alzheimer's patents.

\section{Theoretical frameworks}

Usually when people have memory problems or other symptoms of Alzheimer, they resist against that and deny the symptoms, in these case the family members should realize the symptoms and take the person to the doctor to detect the disease sooner and prevent the progress. We should confess that there is no exact method to detect the disease but a set of methods can help the doctor detection. According to research, a skilled physician can diagnose Alzheimer with accuracy more than 90\%. Although the smart method can diagnose Alzheimer without a doctor but still a doctor is essential for final diagnosis. The diagnosis steps by a psychiatrist or psychologist can be summarized as follows.

\section{Assessment of the mental state}

At this stage, information such as the patient realization from his failures, date, time and place where the he is located, the ability to recall a short list of words or follow specific instructions or to do simple math calculations are collected from the patient. There is a Mini-Mental State Examination (MMSE) test to evaluate the intellectual performance of individuals; in this test some questions are asked from the patient and the answers are scored and based on his total score the disease statues will be diagnosed. The maximum score of MMSE test is 30; score 20-24 indicates mild dementia, 13-20 moderate dementia and score lower than 12 indicate severe dementia. Appendix A is a sample of this test and scoring method.

In addition to assessing the mental state of the patient, the doctor should be able to diagnose other mental diseases that have common symptoms with Alzheimer and cause memory problems in patient and separate them from Alzheimer.

\section{Brain MRI}

Nowadays brain scanning involves structural images such as MRI or CT scans and functional imaging such as PET and FMRI can be a big help in more accurate diagnosis of Alzheimer. Recent structural imaging studies have shown that by disease progresses, the brain of patients significantly depleted and gets small. The researchers also showed that the analysis of a specific area in the brain can detect the early signs of Alzheimer's. However, scientists still could not find an accurate correlation between brain atrophy and Alzheimer progresses over time. The researchers also used functional brain imaging techniques such as PET to diagnose the Alzheimer and follow the progression of disease in the brain and the effect of treatment method on brain function.

\section{Smart detection methods of Alzheimer}

According to the development of intelligent systems in various sciences and their applications, they can also help doctors in automatic detection of diseases. However, given the long history of the discovery of Alzheimer as degenerative brain disease and memory destruction, scientists have conducted many researches for the early detection of this disease and due to advances in biological signal recording and medical imaging devices, and development of processing capabilities, intelligent systems made possible the diagnosis of Alzheimer with high accuracy. Smart Detection Methods of a disease based on brain imaging techniques and recording brain signals (EEG) can be divided into two categories. Methods based on brain imaging were briefly examined in the previous section are not considered here due to their expensive costs and need for special equipment, thus we ignore studying them here. The second categories based on brain signal recording methods are studied here due to their reasonable cost and lack of side effects for the patient.

\section{Review of Literature}

The communicative function of speech was used by Adlai four decades ago (1960), he pioneered in working on theta rhythms of the limbic system of cat brain. The scientists used the functions of speech and vision communication to investigate how the rhythmic potential of the cat brain is associated with its behavior. Using verbal communication in comparing activity of EEG in various brain cells of cats one of the essential steps in dispelling the view that EEG is a secondary symptoms of the disease. The induced theta rhythm and increased work-related communication in the limbic system is a milestone in EEG researches. When we do a 
behavioral task, hippocampus activity shows a transfer of irregular activity to induced rhythms activity of verbal communication. Adler's results (2003) were a decision making factor in the selection of hippocampus as a model for a resonance process in the brain, for Basar research group. Based on numerous tests about cat behavior, Basar assumed that if the brain receives sensory stimulation and if a structure has its own distinct activities, so the structure will respond with its natural frequency. There is some evidence that midline of a region before frontal cortex can generate theta activity in specific cognitive states. These results were reported by Mizuki (1980): EEG rhythms of $5 \mathrm{~Hz}$ frequency became apparent during simple tasks such as counting mentally. Miller (1991) proved that all data are consistent with the hypothesis that theta activity in the frontal areas is associated with theta activity in the hippocampus. Zheng (2005) investigated the EEG verbal communication among and within the hemisphere of the brain at rest and during optic stimulation of the patients with AD. The authors found that the overall decline in AD patients in EEG verbal communication, within the hemisphere, compared to normal resting EEG monitoring, were more significant and more prominent decrease were seen in alpha bands 1 and 2. Adler (2003) studied the EEG verbal communication at rest in $31 \mathrm{AD}$ patients. These authors reported that verbal communication and verbal communication of left temple Alpha and verbal communication of Theta within the hemisphere decreased in AD group.

Locatelli (1998) studied the EEG verbal communication in patients affected by possible AD and they reported that the large relationship between alpha band in AD group decreased compared to the control group. It was more obvious in electrodes of temporal-parietal regions. The decline was more pronounced for verbal communication within the hemisphere of posterior regions. Decrease of Alpha verbal communication was more common in patients with pathological loss. Meanwhile, verbal communication of Theta and Delta within the hemisphere was tended to almost increase in all the analyzed electrodes apart from F7-F8 and T5T6. Locatelli also reported that in electrodes F7-F8 and T5-T6, there is a decrease in verbal communication in delta, theta and alpha bands of AD group compared to control group.

Van D. Hill (2007) investigated the relationship between EEG measures of performance general cognitive tests, memory, language and executive function and realized that there is no difference between alpha-verbal communication of the $\mathrm{AD}$ and control groups and it is not related to cognition. The most common results of all these studies are the reduction of beta and alpha bands verbal communication between separate structures. Zheng Yan (2005) stated that during optical stimulation, EEG verbal communication within and between hemispheres of $\mathrm{AD}$ patients have lower levels of the alpha band than the control group. The authors report that during $5 \mathrm{~Hz}$ optical stimulation, $\mathrm{AD}$ patients had significantly less amounts of verbal communication between the hemispheres in C4-P4 and C3-O1 pairs for theta band, in C3-P3 and C3-O1 and T6-O2 pairs for alpha band and in P3-O1, P4-O2, C3-O1, C4-O2 and T6-O2 pairs for Beta Band.

Hogan (2003) studied the relationship between verbal communication and memory-related EEG power in central and temporal recording locations in early Alzheimer's disease patients and normal control groups. While very average $\mathrm{AD}$ behavioral performance is not significantly different from the normal groups, AD patients decreased the upper alpha verbal communication between the temporal, right and center cortex. Our results are consistent with Hogan findings, although in current study, smaller amounts of alpha-verbal communication, was found only in the untreated group, because in Hogan study the patients were not divided in treated and untreated groups. It should be noted that drugs have effect on alpha verbal communication with long-distance.

\section{Research method \\ Data collection}

FlexComp is a device used to record brain signals. Two channels of $\mathrm{Fz}$ and $\mathrm{Pz}$ are used for recording the signals. Two reference electrodes were attached to the ears and according to international standards 10- 20 active electrodes are determined on the head. Brain signals were taken from individual with open eyes for 2 minutes and 2 minutes blindfolded. There were 10 participants in the test; 6 were normal and 4 had Alzheimer. The average of age of group was above 50 years.

\section{Frequency range}

The power spectrum is calculated by Welch method using a normalized Hanning window for 2 seconds and overlap of $50 \%$ for recorded brain signals for each channel.

\section{Wavelet entropy}

The brain signals are divided into 5 frequency levels by wave entropy. Due to the lack of scales with same length, the coefficients of each level is equal to the number of windows have been divided by one second of 
main signal. In each of the windows respectively the average of wave entropy coefficients energy at each levels of $\mathrm{d} 2$ to $\mathrm{d} 6$ and finally the total energy is calculated. Finally, using the values of energy distribution probability obtained at each level and Shannon entropy with multiplication of values of energy distribution probability at each level with a minus sign in its natural logarithm and adding it for all frequency levels can be calculated.

\section{Lyapunov exponent}

It is a theory that presents geometry and dynamics description of a signal that determines the system order and if it is positive it means that the system is irregular.

$$
\lambda=\frac{1}{n} \ln \frac{d_{n}}{d_{0}}
$$

The method of finding $\square$ is that we find two points in space of path and determine the difference of these two points. Lyapunov fractal is created by mapping the sustainability and chaos areas of point $a \& b$ in sequence of time, for example the yellow part (sustainability) is $\lambda<0$ and blue part (chaos) is $\lambda>0$.

\section{Lyapunov fractal algorithm}

$\lambda=\lim _{N \rightarrow \infty} \frac{1}{N} \sum_{n=1}^{N} \log \left|\frac{d x_{n+1}}{d x_{n}}\right|=\lim _{N \rightarrow \infty} \frac{1}{N} \sum_{n=1}^{N} \log \left|r_{n}\left(1-2 x_{n}\right)\right|$

Fractal is geometrical structure which is made of components which each component is maximized with a certain proportion to make the first structure. In other words, fractal is a structure which each component is similar to whole structure. Fractals are shapes that contrary to Euclidean geometric shapes are not organized in any way. First these shapes are irregular completely, second, the irregularity in all of them is similar and the fractal object is seen identical from far and near, in other words, it is self-similar. Fractals are one of the most important tools in computer graphics but they play the most important role in this new concept of video compression files. The results of fractal and Lyapunov dimension are given in Table 3-11.

Table 1. Participants' features with individual alpha range

\begin{tabular}{|c|c|c|c|c|c|c|c|c|}
\hline Iaf & Alpha high range & Alpha low range & Person's code & Person's condition & age & Last name & First name & row \\
\hline 10.2450 & 12.5000 & 8.0000 & 1 & sick & 60 & abareshi & Zahra & 1 \\
\hline 9.7000 & 12.0000 & 8.0000 & 0 & healthy & 55 & babania & maryam & 2 \\
\hline 10.6000 & 13.5000 & 7.0000 & 0 & healthy & 55 & babania & ali & 3 \\
\hline 9.7000 & 12.5000 & 7.0000 & 1 & sick & 58 & farshadi & mansureh & 4 \\
\hline 9.4700 & 12.0000 & 7.0000 & 0 & healthy & 50 & sadegh pour & Parvin & 5 \\
\hline 9.4000 & 12.5000 & 7.0000 & 0 & healthy & 30 & sedghi & pejman & 6 \\
\hline 10.3100 & 13.0000 & 8.5000 & 0 & healthy & 58 & sedghi & sara & 7 \\
\hline 7.9800 & 10.0000 & 6.0000 & 1 & sick & 60 & yousefi & zohreh & 8 \\
\hline 10.4900 & 13.0000 & 8.0000 & 0 & healthy & 60 & naseri & Ali & 9 \\
\hline 10.9137 & 12.0000 & 8.0000 & 1 & sick & 65 & soltanifar & Hasan & 10 \\
\hline
\end{tabular}

Table 2. Frequency features related to the brain waves (calculation of power and energy spectrum)

\begin{tabular}{|c|c|c|c|c|c|c|c|c|c|}
\hline \multicolumn{3}{|c|}{ f open } & \multicolumn{5}{c|}{ f close } \\
\hline Gama & beta & alpha & theta & Delta & Gama & beta & alpha & theta & Delta \\
\hline 34.8386 & 41.1076 & 28.6853 & 24.9289 & 57.5434 & 36.3032 & 55.5266 & 165.9348 & 26.6890 & 20.3648 \\
\hline
\end{tabular}




\begin{tabular}{|l|c|c|c|c|c|c|c|c|c|}
\hline 18.5250 & 19.7253 & 47.2474 & 19.2225 & 23.7729 & 21.4751 & 23.9920 & 77.0244 & 18.2651 & 27.2976 \\
\hline 6.5987 & 8.1007 & 7.4190 & 14.6517 & 25.6559 & 12.5590 & 11.8493 & 37.4300 & 17.5759 & 39.9226 \\
\hline 20.6735 & 34.1214 & 37.4883 & 32.0841 & 54.3805 & 17.8962 & 29.7352 & 61.3142 & 21.3761 & 27.8875 \\
\hline 27.2668 & 50.6267 & 83.3150 & 48.8559 & 42.9864 & 17.4305 & 49.6491 & 127.9188 & 52.7064 & 34.0531 \\
\hline 12.5411 & 11.2746 & 24.7493 & 24.7629 & 56.0748 & 10.1709 & 14.9897 & 44.3771 & 45.7211 & 97.1984 \\
\hline 15.4638 & 17.0632 & 14.5162 & 18.9895 & 67.5189 & 12.4665 & 15.1617 & 21.3153 & 21.7571 & 45.5004 \\
\hline 7.5082 & 13.7435 & 60.6196 & 35.0109 & 14.2776 & 7.3816 & 18.7000 & 65.9802 & 42.6901 & 18.2426 \\
\hline 10.1272 & 7.1550 & 8.0960 & 8.8403 & 13.9832 & 11.6122 & 7.2398 & 12.8490 & 10.8790 & 16.5401 \\
\hline 13.0989 & 14.1634 & 7.5879 & 8.9236 & 24.0970 & 15.2957 & 19.4000 & 8.2684 & 9.7431 & 24.4937 \\
\hline
\end{tabular}

Table 3. Frequency features related to the brain waves (calculation of power and energy spectrum)

\begin{tabular}{|c|c|c|c|c|c|c|c|c|c|}
\hline \multicolumn{7}{|c|}{ Frequency features Fz } \\
\hline \multicolumn{7}{|c|}{ rf open } & \multicolumn{5}{c|}{ rf close } \\
\hline Gama & beta & alpha & theta & delta & Gama & beta & alpha & theta & delta \\
\hline 0.1940 & 0.2289 & 0.1597 & 0.1388 & 0.3204 & 0.1251 & 0.1913 & 0.5718 & 0.0920 & 0.0702 \\
\hline 0.1388 & 0.1477 & 0.3539 & 0.1440 & 0.1781 & 0.1256 & 0.1403 & 0.4505 & 0.1086 & 0.1597 \\
\hline 0.0706 & 0.0867 & 0.0794 & 0.1568 & 0.2746 & 0.0944 & 0.0891 & 0.2814 & 0.1321 & 0.3001 \\
\hline 0.1180 & 0.1948 & 0.2140 & 0.1832 & 0.3104 & 0.1160 & 0.1927 & 0.3974 & 0.1385 & 0.1807 \\
\hline 0.1063 & 0.1974 & 0.3326 & 0.1944 & 0.1676 & 0.0644 & 0.1835 & 0.4727 & 0.1948 & 0.1258 \\
\hline 0.0935 & 0.0841 & 0.1845 & 0.1846 & 0.4180 & 0.0486 & 0.0716 & 0.2121 & 0.2185 & 0.4646 \\
\hline 0.1106 & 0.1221 & 0.1038 & 0.1358 & 0.4830 & 0.1081 & 0.1314 & 0.1848 & 0.1886 & 0.3945 \\
\hline 0.0617 & 0.1129 & 0.4980 & 0.2876 & 0.1173 & 0.0516 & 0.1307 & 0.4613 & 0.2985 & 0.1275 \\
\hline 0.1179 & 0.0833 & 0.0943 & 0.1029 & 0.1628 & 0.1538 & 0.0959 & 0.1702 & 0.1441 & 0.2190 \\
\hline 0.1990 & 0.2152 & 0.1153 & 0.1356 & 0.3661 & 0.2066 & 0.2620 & 0.1117 & 0.1316 & 0.3308 \\
\hline
\end{tabular}

Table 4. Frequency features related to the brain waves (calculation of power and energy spectrum)

\begin{tabular}{|c|c|c|c|c|c|c|c|c|c|}
\hline \multicolumn{7}{|c|}{ frequency features Oz } \\
\hline Gama & beta & alpha & theta & delta & Gama & beta & alpha & theta & delta \\
\hline 14.0542 & 21.6330 & 25.8208 & 32.5990 & 27.8291 & 18.0374 & 31.3463 & 118.4254 & 28.8005 & 20.1194 \\
\hline 56.7676 & 20.4027 & 34.5516 & 9.3660 & 7.5793 & 53.5344 & 24.3995 & 107.8586 & 11.5504 & 6.7896 \\
\hline 17.6601 & 18.5115 & 15.3506 & 19.6768 & 16.7522 & 18.4343 & 23.5217 & 43.6135 & 21.2731 & 12.9922 \\
\hline
\end{tabular}




\begin{tabular}{|l|c|c|c|c|c|c|c|c|c|}
12.7082 & 22.2228 & 32.6496 & 25.5680 & 31.2369 & 7.0869 & 18.5341 & 60.4566 & 19.5166 & 28.7821 \\
\hline 32.6484 & 69.6886 & 116.3989 & 40.4695 & 24.8874 & 18.4337 & 71.7480 & 212.0194 & 46.2392 & 22.2571 \\
\hline 36.9690 & 23.9271 & 44.2069 & 25.3160 & 41.1345 & 27.7598 & 26.3276 & 85.3345 & 45.4925 & 45.9330 \\
\hline 32.6849 & 24.8688 & 19.9081 & 13.0786 & 60.3481 & 26.4550 & 26.1572 & 50.2229 & 14.0871 & 27.2944 \\
\hline 13.9957 & 18.8956 & 20.1217 & 11.9083 & 7.7592 & 10.7467 & 16.1400 & 61.5107 & 11.5284 & 9.2951 \\
\hline 17.2904 & 9.1713 & 9.9884 & 6.5021 & 7.1125 & 13.0839 & 7.8329 & 10.8258 & 6.5169 & 6.5114 \\
\hline 34.5773 & 11.1228 & 5.8068 & 7.1926 & 11.7080 & 29.8331 & 12.6292 & 6.2414 & 6.8618 & 13.7249 \\
\hline
\end{tabular}

Table 5. Frequency features related to the brain waves (calculation of power and energy spectrum)

\begin{tabular}{|c|c|c|c|c|c|c|c|c|c|}
\hline \multicolumn{7}{|c|}{ Frequency features Oz } \\
\hline rf open & \multicolumn{7}{c|}{ rf close } \\
\hline Gama & beta & alpha & theta & delta & Gama & beta & alpha & theta & delta \\
\hline 0.1202 & 0.1850 & 0.2208 & 0.2887 & 0.2379 & 0.0863 & 0.1500 & 0.5668 & 0.1378 & 0.0963 \\
\hline 0.3499 & 0.1258 & 0.2130 & 0.0495 & 0.0467 & 0.2296 & 0.1046 & 0.4626 & 0.0577 & 0.0291 \\
\hline 0.1251 & 0.1312 & 0.1088 & 0.1394 & 0.1187 & 0.1074 & 0.1371 & 0.2542 & 0.1240 & 0.0757 \\
\hline 0.1040 & 0.1819 & 0.2672 & 0.2093 & 0.2557 & 0.0540 & 0.1413 & 0.4608 & 0.1487 & 0.2194 \\
\hline 0.1170 & 0.2498 & 0.4172 & 0.1450 & 0.0892 & 0.0512 & 0.1993 & 0.5889 & 0.1284 & 0.0618 \\
\hline 0.1988 & 0.1317 & 0.2433 & 0.1393 & 0.2263 & 0.1189 & 0.1127 & 0.3654 & 0.1948 & 0.1967 \\
\hline
\end{tabular}

Table 6. Features related to the spectrum of the brain signals conformity

\begin{tabular}{|c|c|c|c|c|c|c|c|c|c|}
\hline \multicolumn{5}{|c|}{ coherence open with open } & \multicolumn{4}{c|}{ coherence close with close } \\
\hline Gama & Beta & Alpha & Theta & Delta & Gama & Beta & Alpha & Theta & Delta \\
\hline 0.6086 & 0.1231 & 0.0945 & 0.1056 & 0.0788 & 0.4457 & 0.1257 & 0.0456 & 0.0649 & 0.0788 \\
\hline 2.1169 & 0.2515 & 0.1832 & 0.1938 & 0.1289 & 9.3729 & 0.4424 & 0.7630 & 0.2695 & 0.5708 \\
\hline 14.6753 & 6.1255 & 2.8229 & 1.9504 & 2.2691 & 3.5332 & 0.8665 & 0.7717 & 0.1814 & 0.6441 \\
\hline 9.0870 & 3.0135 & 2.6290 & 2.3097 & 0.9581 & 19.5609 & 4.5520 & 4.6327 & 2.5838 & 2.3479 \\
\hline 0.6128 & 0.2262 & 0.1229 & 0.1037 & 0.1486 & 0.6143 & 0.2035 & 0.1598 & 0.1164 & 0.0767 \\
\hline 0.7836 & 0.1212 & 0.1364 & 0.0716 & 0.0766 & 0.2647 & 0.1981 & 0.1166 & 0.1102 & 0.1005 \\
\hline 0.5058 & 0.2397 & 0.1334 & 0.1222 & 0.1037 & 11.3014 & 0.4537 & 0.4812 & 1.2505 & 2.6593 \\
\hline 4.5958 & 0.7285 & 0.2884 & 0.3240 & 0.7190 & 1.7514 & 0.4882 & 0.3286 & 0.1552 & 0.1914 \\
\hline 1.8107 & 0.3614 & 0.1492 & 0.1692 & 0.2642 & 6.0413 & 0.2961 & 0.3225 & 0.2312 & 0.2709 \\
\hline 19.7499 & 4.3134 & 2.8288 & 4.0037 & 4.7548 & 16.0870 & 2.7901 & 2.5590 & 2.8252 & 4.1054 \\
\hline
\end{tabular}


Table 7. Features of Violet waves

\begin{tabular}{|c|c|c|c|c|c|c|c|c|c|}
\hline \multicolumn{10}{|c|}{ Wavelet } \\
\hline \multicolumn{5}{|c|}{ sig open $\mathrm{fz}$} & \multicolumn{5}{|c|}{ sig close $\mathrm{fz}$} \\
\hline Gama & beta & alpha & theta & delta & Gama & beta & alpha & theta & delta \\
\hline-0.0031 & -0.0310 & 0.1346 & -0.4213 & -0.3033 & -0.0041 & 0.1006 & -0.1435 & -0.3154 & -0.0410 \\
\hline 0.0023 & -0.0409 & 0.1940 & 0.5202 & -0.2032 & $1.3657 \mathrm{e}-04$ & 0.0223 & -0.0303 & 0.4227 & -0.9020 \\
\hline$-1.3135 \mathrm{e}-04$ & -0.0647 & 0.0532 & -0.0828 & -0.0526 & $-1.4289 \mathrm{e}-04$ & 0.0141 & 0.0060 & -0.2446 & 0.0304 \\
\hline-0.0015 & -0.0536 & -0.2810 & 0.1196 & -0.2051 & $1.2186 \mathrm{e}-04$ & -0.0324 & -0.2535 & -0.3684 & -0.1219 \\
\hline 0.0099 & -0.0922 & 0.0724 & -0.0262 & -0.1865 & -0.0015 & -0.0945 & 0.5202 & 0.5107 & -0.0863 \\
\hline-0.0051 & -0.1471 & -0.0278 & 0.2406 & -0.2553 & 0.0031 & 0.0219 & -0.1477 & -0.2047 & -0.2452 \\
\hline-0.0041 & -0.0601 & 0.3734 & -0.1343 & -0.4618 & $2.5677 \mathrm{e}-04$ & 0.0660 & 0.1153 & -0.3460 & -0.0782 \\
\hline 0.0034 & -0.0576 & 0.0315 & 0.0763 & -0.1890 & -0.0014 & -0.0901 & 0.0202 & 0.3866 & -0.2667 \\
\hline$-6.7275 \mathrm{e}-04$ & -0.0506 & -0.0622 & -0.1286 & -0.1332 & $-2.4443 e-04$ & 0.0029 & -0.0055 & -0.1041 & -0.0437 \\
\hline $5.3065 \mathrm{e}-05$ & 0.0042 & 0.0086 & 0.1368 & -0.1016 & $1.4470 \mathrm{e}-04$ & -0.0656 & 0.2498 & 0.1679 & -.1164 \\
\hline
\end{tabular}

Table 8. Features related to Violet waves

\begin{tabular}{|c|c|c|c|c|c|c|c|c|c|}
\hline \multicolumn{7}{|c|}{ Sig open oz } & \multicolumn{3}{c|}{ sig close oz } \\
\hline Gama & beta & alpha & theta & delta & Gama & beta & alpha & theta & delta \\
\hline $1.7438 \mathrm{e}-04$ & 0.0083 & 0.0665 & -0.0533 & 0.0647 & -0.0034 & -0.1137 & 0.3099 & 0.3400 & 0.0572 \\
\hline 0.0010 & 0.3328 & -0.0997 & 0.0694 & -0.0488 & $-2.7407 \mathrm{e}-04$ & -0.0432 & -0.1407 & 0.3045 & -0.0460 \\
\hline$-4.7210 \mathrm{e}-04$ & -0.1005 & 0.0169 & 0.0987 & -0.0540 & $-4.2435 \mathrm{e}-04$ & $-7.7196 \mathrm{e}-04$ & 0.2099 & 0.0334 & -0.0673 \\
\hline$-5.3340 \mathrm{e}-04$ & 0.0513 & -0.0582 & -0.3212 & -0.0187 & $9.9460 \mathrm{e}-04$ & -0.0729 & -0.4062 & -0.2568 & 0.0217 \\
\hline $1.1783 \mathrm{e}-04$ & 0.1263 & 0.3529 & 0.5130 & -0.0646 & 0.0050 & -0.0391 & -0.3474 & -0.5707 & 0.0823 \\
\hline$-2.5577 \mathrm{e}-04$ & 0.0098 & 0.0808 & -0.1911 & -0.0215 & 0.0011 & 0.0814 & -0.0386 & -1.0157 & -0.1004 \\
\hline-0.0011 & 0.1430 & $3.9392 \mathrm{e}-04$ & 0.1377 & 0.4471 & $5.1332 \mathrm{e}-04$ & -0.0729 & -0.0035 & 0.0808 & -0.0477 \\
\hline$-3.4415 \mathrm{e}-04$ & -0.0299 & 0.0308 & 0.0904 & -0.0386 & $7.2484 \mathrm{e}-04$ & 0.0555 & -0.0305 & 0.0780 & -0.0221 \\
\hline$-2.2693 \mathrm{e}-04$ & -0.1447 & 0.0197 & 0.0302 & -0.0522 & $-5.5022 \mathrm{e}-04$ & 0.0485 & 0.1719 & -0.1992 & -0.0423 \\
\hline$-6.8460 \mathrm{e}-04$ & -0.1866 & 0.0667 & -0.0093 & 0.0202 & $-1.9132 \mathrm{e}-04$ & 0.0922 & 0.0221 & 0.3697 & 0.0021 \\
\hline
\end{tabular}


Table 9. Features related to entropy violet waves

\begin{tabular}{|c|c|c|c|c|c|c|c|c|c|c|c|}
\hline \multicolumn{9}{|c|}{ save entropy } \\
\hline \multicolumn{3}{|c|}{ sig open fz } & \multicolumn{7}{c|}{ sig close fz } \\
\hline swt & Gama & beta & alpha & theta & delta & Swt & Gama & beta & alpha & theta & delta \\
\hline 1.3457 & 0.1057 & 0.3466 & 0.3213 & 0.1489 & 0.0775 & 0.9958 & 0.0498 & 0.2200 & 0.6301 & 0.0826 & 0.0175 \\
\hline 1.3007 & 0.1304 & 0.2421 & 0.4243 & 0.1592 & 0.0440 & 1.2305 & 0.0993 & 0.2199 & 0.5047 & 0.1378 & 0.0383 \\
\hline 1.4380 & 0.3264 & 0.1578 & 0.2200 & 0.2060 & 0.0897 & 1.3228 & 0.1676 & 0.1429 & 0.4260 & 0.1784 & 0.0851 \\
\hline 1.3757 & 0.0892 & 0.2948 & 0.3428 & 0.1880 & 0.0852 & 1.2238 & 0.0593 & 0.2383 & 0.5062 & 0.1456 & 0.0507 \\
\hline 1.3031 & 0.0894 & 0.2047 & 0.4466 & 0.2052 & 0.0540 & 1.1418 & 0.0410 & 0.1648 & 0.5507 & 0.2023 & 0.0412 \\
\hline 1.4324 & 0.1451 & 0.1551 & 0.3430 & 0.2283 & 0.1285 & 1.3446 & 0.0725 & 0.1064 & 0.3942 & 0.2710 & 0.1559 \\
\hline 1.4685 & 0.1490 & 0.2555 & 0.2542 & 0.2127 & 0.1285 & 1.4150 & 0.1028 & 0.2287 & 0.3377 & 0.2235 & 0.1073 \\
\hline 1.1476 & 0.0533 & 0.0934 & 0.3957 & 0.4084 & 0.0492 & 1.1283 & 0.0434 & 0.0894 & 0.4368 & 0.3718 & 0.0586 \\
\hline 1.3272 & 0.4491 & 0.1852 & 0.1877 & 0.1310 & 0.0470 & 1.4197 & 0.2947 & 0.2064 & 0.2841 & 0.1604 & 0.0544 \\
\hline 1.3607 & 0.0935 & 0.3880 & 0.2576 & 0.1648 & 0.0960 & 1.3637 & 0.0826 & 0.4205 & 0.2417 & 0.1599 & 0.0953 \\
\hline
\end{tabular}

Table 10. Features related to entropy violet waves

\begin{tabular}{|c|c|c|c|c|c|c|c|c|c|c|c|}
\hline \multicolumn{9}{|c|}{ wave entropy } \\
\hline \multicolumn{3}{|c|}{ sig open oz } & \multicolumn{7}{c|}{ sig close oz } \\
\hline swt & Gama & beta & alpha & theta & delta & Swt & Gama & beta & alpha & theta & delta \\
\hline 1.3586 & 0.0865 & 0.2283 & 0.3658 & 0.2587 & 0.0606 & 1.0178 & 0.0472 & 0.1644 & 0.6384 & 0.1268 & 0.0233 \\
\hline 1.2720 & 0.3763 & 0.2716 & 0.2599 & 0.0758 & 0.0164 & 1.1911 & 0.2278 & 0.2153 & 0.4807 & 0.0649 & 0.0113 \\
\hline 1.4010 & 0.3591 & 0.1997 & 0.2467 & 0.1572 & 0.0374 & 1.3113 & 0.2834 & 0.1713 & 0.3860 & 0.1327 & 0.0267 \\
\hline 1.3841 & 0.1012 & 0.2368 & 0.3748 & 0.2118 & 0.0755 & 1.1466 & 0.0389 & 0.1683 & 0.5704 & 0.1599 & 0.0625 \\
\hline 1.2180 & 0.0902 & 0.2178 & 0.5038 & 0.1552 & 0.0329 & 0.9991 & 0.0317 & 0.1644 & 0.6350 & 0.1453 & 0.0237 \\
\hline 1.4130 & 0.2301 & 0.1951 & 0.3564 & 0.1479 & 0.0705 & 1.2953 & 0.1250 & 0.1383 & 0.4791 & 0.1984 & 0.0592 \\
\hline 1.4390 & 0.2337 & 0.2971 & 0.2518 & 0.1248 & 0.0927 & 1.3066 & 0.1640 & 0.2752 & 0.4118 & 0.1041 & 0.0449 \\
\hline 1.3924 & 0.1722 & 0.2230 & 0.3451 & 0.2198 & 0.0399 & 1.1482 & 0.0879 & 0.1231 & 0.5207 & 0.2413 & 0.0270 \\
\hline 1.3393 & 0.3703 & 0.2604 & 0.2360 & 0.1019 & 0.0315 & 1.3750 & 0.2913 & 0.2474 & 0.3012 & 0.1285 & 0.0316 \\
\hline 1.3268 & 0.3767 & 0.3308 & 0.1528 & 0.1011 & 0.0386 & 1.3516 & 0.3282 & 0.3528 & 0.1785 & 0.0950 & 0.0455 \\
\hline
\end{tabular}


Table 11. Features related to fractal dimension and Lyapunov exponent of the brain waves

\begin{tabular}{|c|c|c|c|c|c|c|l|}
\hline \multicolumn{5}{|c|}{} & \multicolumn{4}{c|}{ Liayapunov } \\
\hline sig close fz & sig open fz & sig close oz & sig open oz & sig close fz & sig open fz & sig close oz & sig open oz \\
\hline 1.0101 & 1.0114 & 1.0103 & 1.0119 & 0.0556 & 0.5001 & 0.3351 & 0.6670 \\
\hline 1.0129 & 1.0129 & 1.0130 & 1.0140 & 0.5778 & 0.5090 & 0.7715 & 0.7388 \\
\hline 1.0121 & 1.0127 & 1.0137 & 1.0149 & 0.4810 & 0.7852 & 0.0900 & 0.8628 \\
\hline 1.0109 & 1.0117 & 1.0103 & 1.0121 & 0.6801 & 0.6160 & 0.5867 & 0.6608 \\
\hline 1.0103 & 1.0122 & 1.0082 & 1.0113 & 0.0019 & 0.2001 & 0.0019 & 0.0011 \\
\hline 1.0121 & 1.0139 & 1.0131 & 1.0146 & 0.1937 & 0.5512 & 0.0687 & 0.6376 \\
\hline 1.0121 & 1.0131 & 1.0129 & 1.0140 & 0.5462 & 0.6655 & 0.7003 & 0.6265 \\
\hline 1.0109 & 1.0110 & 1.0126 & 1.0136 & 0.5530 & 0.5650 & 0.7722 & 0.8338 \\
\hline 1.0132 & 1.0132 & 1.0143 & 1.0148 & 0.7694 & 0.8499 & 0.8434 & 0.8631 \\
\hline 1.0113 & 1.0116 & 1.0141 & 1.0146 & 0.7312 & 0.7288 & 0.8755 & 0.8408 \\
\hline
\end{tabular}

\section{Findings}

SPSS software and t-test analyze were used to conclude that the following 5 features separate normal people from mild Alzheimer patients in way that we used 0 for healthy people and 1 for mild Alzheimer patients and the results are presented in table 4-1 and 4-2.

Table 4-1 the results of data analysis

\begin{tabular}{|c|c|}
\hline & Function \\
\cline { 2 - 2 } & 1 \\
\hline Delta rf open oz & -15.855 \\
Beta coherence close with close & 3.692 \\
Wavelet Alpha sig close fz & -53.550 \\
Wavelet Teta sig open oz & 106.379 \\
FD sig open fz & 23938.680 \\
(Constant) & -24238.091 \\
\hline
\end{tabular}

Table 4-2: Separating healthy people from the patients

\begin{tabular}{|c|c|c|c|c|}
\hline & \multirow[t]{2}{*}{ Patient } & \multicolumn{2}{|c|}{ Predicted Group Membership } & \multirow[b]{2}{*}{ Total } \\
\hline & & 0 & 1 & \\
\hline \multirow[t]{2}{*}{ Original } & 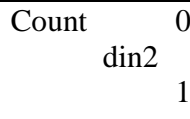 & $\begin{array}{l}6 \\
0\end{array}$ & $\begin{array}{l}0 \\
4\end{array}$ & $\begin{array}{l}6 \\
4\end{array}$ \\
\hline & $\% \quad \begin{array}{l}0 \\
\\
\end{array}$ & $\begin{array}{c}100.0 \\
.0\end{array}$ & $\begin{array}{c}.0 \\
100.0\end{array}$ & $\begin{array}{l}100.0 \\
100.0\end{array}$ \\
\hline
\end{tabular}




\begin{tabular}{|c|c|c|c|c|}
\hline Cross-validateda & $\begin{array}{l}\text { Count } \\
\\
\end{array}$ & $\begin{array}{l}6 \\
0\end{array}$ & $\begin{array}{l}0 \\
4\end{array}$ & $\begin{array}{l}6 \\
4\end{array}$ \\
\hline & $\% \quad \operatorname{dim} 2{ }_{1}^{0}$ & $\begin{array}{c}100.0 \\
.0\end{array}$ & $\begin{array}{c}.0 \\
100.0\end{array}$ & $\begin{array}{l}100.0 \\
100.0\end{array}$ \\
\hline
\end{tabular}

\section{Conclusion}

FlexComp is a device used to record brain signals. Two channels of $\mathrm{Fz}$ and $\mathrm{Pz}$ are used for the reference of electrode ear to record the signals. Brain signals were taken from individual with open eyes for 2 minutes and 2 minutes blindfolded. Best results were extracted in delta rf open oz bbeta coherence close with close ، wavelet Alpha sig close $\mathrm{fz}$ ،wavelet theta sig open oz 9 FD sig open fz modes.

\section{References}

Adler, G., Brassen, S., Jajcevic, A., 2003. EEG coherence in Alzheimer's dementia. J. Neural. Transm. 110, 1051-1058.

Chapman, Robert M., et al. "Brain event-related potentials: diagnosing early-stage Alzheimer's disease." Neurobiology of aging 28.2 (2007): 194-201.

Güntekin, Bahar, Ertuğrul Saatçi, and Görsev Yener. "Decrease of evoked delta, theta and alpha coherences in Alzheimer patients during a visual oddball paradigm." Brain research 1235 (2008): 109-116.

Hogan, M.J., Swanwick, G.R.J., Kaiser, J., Rowan, M., Lawlor, B., 2003. Memory-related EEG power and coherence reductions in mild Alzheimer's disease. Int. J. Psychophysiol. 49, 147-163.

Locatelli, T., Cursi, M., Liberati, D., Franceschi, M., Comi, G., 1998. EEG coherence in Alzheimers disease. Electroencephalogr, Clin. Neurophys. 106, 229-237.

Miller, R., 1991. Cortico-Hippocampal Interplay and the Representation of Contexts in the Brain. Springer, Berlin. Miltner, W., Braun, C., Arnold, M., Witte, H., Taub, E., 1999. Coherence of gamma-band EEG activity as a basis for associative learning. Nature 397, 434-436.

Mizuki, Y., Masotoshi, T., Isozaki, H., Nishijima, H., Inanaga, K., 1980. Periodic appearance of theta rhythm in the frontal midline area during performance of a mental task Electroencephalogr. Clin. Neurophysiol. $49,345-351$.

van der Hiele, K., Vein, A.A., Reijntjes, R.H.A.M., Westendorp, R.G. J., Bollen, E.L.E.M., van Buchem, M.A., van Dijk, J.G., Middelkoop, H.A.M., 2007. EEG correlates in the spectrum of cognitive decline. Clin. Neurophysiol. 118, 1931-1939.

Zheng-yan, J., 2005. Abnormal cortical functional connections in Alzheimer's disease: analysis of inter- and intra-hemispheric EEG coherence. J. Zhejiang Univ. SCI. 6B (4), 259-264. 